\title{
Long-term erosion of plasma-facing materials with different surface roughness in ASDEX Upgrade
}

\author{
A Hakola ${ }^{1}$, J Karhunen ${ }^{1}$, S Koivuranta ${ }^{1}$, J Likonen ${ }^{1}$, M Balden ${ }^{2}$, A Herrmann ${ }^{2}$, M Mayer², H W \\ Müller $^{2}$, R Neu ${ }^{2}$, V Rohde ${ }^{2}$, K Sugiyama ${ }^{2}$ and the ASDEX Upgrade team \\ ${ }^{1}$ VTT, Association EURATOM-Tekes, P O Box 1000, 02044 VTT, Finland \\ ${ }^{2}$ Max-Planck-Institut für Plasmaphysik, EURATOM Association, Boltzmannstr.2, 85748 Garching, \\ Germany
}

E-mail: antti.hakola@vtt.fi

\begin{abstract}
The effect of surface roughness on the long-term erosion patterns of tungsten coatings was investigated in the outer strike-point region of ASDEX Upgrade during its 2010/11 plasma operations. The net erosion rates of rough coatings $\left(R_{\mathrm{a}}=5-6 \mu \mathrm{m}\right)$ were 3-7 times smaller than those of smooth coatings $\left(R_{\mathrm{a}}=0.4-0.8\right.$ $\mu \mathrm{m})$. This is because rough surfaces are largely modified and damaged in the microscopic scale but the material is re-deposited together with boron, deuterium, and carbon on the shadowed sides of the most prominent surface features. In addition, we observed that $\mathrm{W}$ coatings were eroded on average at a rate of $0.03 \mathrm{~nm} / \mathrm{s}$, which was 3-4 times smaller than the value for $\mathrm{Cr}$, simulating here steel.
\end{abstract}

PACS: $52.40 . \mathrm{Hf}$, 52.55.Fa, 82.80.Ms, 82.80.Yc

\section{Introduction}

Tungsten (W) is an actively studied candidate material for the first wall of future fusion reactors, mainly due to its small erosion yield by physical sputtering but also because retention of plasma fuel in W-based plasma-facing components (PFCs) is low [1,2]. Under reactor conditions, however, the material properties of $\mathrm{W}$ can be severely degraded and intense power and particle loads may seriously damage $\mathrm{W}$ surfaces. Moreover, even small amounts of $\mathrm{W}$ transported into the core plasma can restrict its performance via increased radiation losses $[1,3]$. More research on the behaviour of $\mathrm{W}$ in different operational regimes of a reactor is therefore needed.

One important goal is to properly understand the erosion characteristics of tungsten PFCs during longterm plasma operations. We have studied this issue in ASDEX Upgrade (AUG), which has been operated with full-W first wall since 2007 [4]. Our investigations have been concentrated on the outer strike-point (OSP) region, expected to be the main source of W in AUG, where we have exposed graphite tiles with specific marker coatings to plasma discharges during experimental campaigns of 3000-6000 s with divertor plasmas.

In this article, we report our results on the effect of surface roughness on the net erosion rate of tungsten and compare the erosion behaviour of $\mathrm{W}$ with that of $\mathrm{Mo}, \mathrm{Cr}$, and $\mathrm{Al}$; here, $\mathrm{Cr}$ simulates steel while $\mathrm{Al}$ is used as a proxy for beryllium. The results have been obtained from marker tiles exposed to plasma during the 2010/11 experimental campaign of AUG. The erosion profiles of the tiles have been 
measured using various ion-beam methods and the surface morphology has been studied using scanning electron microscopy (SEM) and confocal laser-scanning microscopy (CLSM).

\section{Experimental details}

\subsection{Marker tiles in 2010/11}

For the 2010/11 campaign, DIARC-Technology Inc. coated three graphite OSP tiles with marker stripes. The first two tiles, Bgr 1-1 and 1-2, originated from the toroidal sectors 11 and 2 of AUG, respectively, and had three equally wide poloidal stripes of different surface roughness (produced by glass-pearl blasting) and a $2-\mu \mathrm{m}$ thick $\mathrm{W}$ coating. The original arithmetic average roughnesses, $R_{\mathrm{a}}$, of the uncoated stripes were $5.5 \mu \mathrm{m}$ (W-r1), $0.8 \mu \mathrm{m}$ (W-r2), and $0.4 \mu \mathrm{m}$ (W-r3). The third tile (Bgr 1-3, from sector 11) was not blasted, only coated with four equally wide, 3-4 $\mu \mathrm{m}$ thick poloidal $\mathrm{Al}, \mathrm{Cr}$, Mo, and $\mathrm{W}$ marker stripes. The surface roughness of the tile was not measured but based on earlier results, the average $R_{\mathrm{a}}$ value of the graphite surface is around $1.5 \mu \mathrm{m}$ [5]. The poloidal location of the tiles in the divertor as well as reference values for the $s$ coordinate, running along the tile surfaces, are marked in the inset of Figure 1(a).

The total discharge time of the 2010/11 campaign was $5693.5 \mathrm{~s}$ in the lower single-null configuration, consisting of 1023 plasma discharges. In addition, 7 boronizations were carried out during the campaign and the OSP was mainly located in the Bgr 1 tiles as the strike-point distribution in Figure 1(a) indicates.

\subsection{Post mortem analyses}

All the tiles were analyzed using Rutherford Backscattering Spectroscopy (RBS) before and after the 2010/11 campaign. We used a 3.0-MeV proton beam in the analyses and detected the backscattered particles at a scattering angle of $165^{\circ}$. The diameter of the beam was approximately $1.8 \mathrm{~mm}$. By fitting the recorded proton spectra with the SIMNRA program, the elemental composition and thickness of each marker layer were determined.

In addition to the erosion studies, we measured the deposition of deuterium, boron, and carbon on the marker stripes using Nuclear Reaction Analysis (NRA). To this end, a beam of ${ }^{3} \mathrm{He}^{+}$ions with an energy of $2.5 \mathrm{MeV}$ was used, and the protons formed in the nuclear reactions $\mathrm{D}\left({ }^{3} \mathrm{He}, \mathrm{p}\right){ }^{4} \mathrm{He}$, ${ }^{11} \mathrm{~B}\left({ }^{3} \mathrm{He}, \mathrm{p}\right){ }^{13} \mathrm{C}$, and ${ }^{12} \mathrm{C}\left({ }^{3} \mathrm{He}, \mathrm{p}\right){ }^{14} \mathrm{~N}$ were recorded at $150^{\circ}$.

Depth profiles of different elements on the marker coatings were measured by Secondary Ion Mass Spectrometry (SIMS). The analyses were carried out using a VG Ionex IX-70S double-focusing magnetic sector instrument with a $5-\mathrm{keV} \mathrm{O}_{2}{ }^{+}$primary ion beam and with a current of $500 \mathrm{nA} \mathrm{[6].} \mathrm{In}$ the measurements, the beam was raster-scanned across an area of $0.3 \times 0.4 \mathrm{~mm}^{2}$.

The surface topography of selected tiles was investigated by SEM (FEI XL30 ESEM) using both secondary and backscattered electron detectors. The roughness of the surface was determined by CLSM (Olympus LEXT OSL4000).

\section{Results}

\subsection{Erosion/deposition profiles}

A large surface roughness leads to reduced net erosion of $\mathrm{W}$ compared to the case of smooth coatings. This is illustrated in Figure 1 where the poloidal net erosion profiles for the different $\mathrm{W}$ stripes on the 2010/11 marker tiles are presented (Figure 1(b)) together with poloidal re-deposition curves on the Mo and $\mathrm{Cr}$ markers of the tile Bgr 1-3 (Figure 1(c)). Especially, the data from the tiles Bgr 1-2 and 1-3 show that the maximum net erosion consistently decreases with roughness; it is approximately $3.5 \times 10^{18} \mathrm{at} / \mathrm{cm}^{2}$ for the smoothest (W-r3) stripe, $1.1 \times 10^{18} \mathrm{at} / \mathrm{cm}^{2}$ for the reference W stripe, and $0.04 \times 10^{18} \mathrm{at} / \mathrm{cm}^{2}$ for the roughest (W-r1) stripe around $\mathrm{s} \approx 1120 \mathrm{~mm}$. In addition, one observes that the toroidally integrated re-deposition of $\mathrm{W}$ atoms $\left(\sim 1.0 \times 10^{22}\right)$ is comparable to the overall net erosion of 
the reference coatings $\left(\sim 1.4 \times 10^{22}\right.$ atoms, tile Bgr $\left.1-3\right)$; these numbers have been obtained by multiplying the average erosion/deposition data in Figures 1(b) and (c) by the corresponding toroidal length and the poloidal distance between successive measurement points throughout the tile. This means that the role of re-deposition becomes more prominent as the roughness of the coating increases.

The erosion maximum is typically reached around $s \approx 1075-1125 \mathrm{~mm}$ except for the W-r3 stripe on the tile Bgr 1-1 for which a distinguishable net deposition peak is observed in the OSP region. This is attributed to the fact that the particular stripe was more shadowed from plasma contact during the entire campaign than the other stripes, as discussed in Ref. [7]. The shadowing effect plays the largest role close to the upstream edge of the Bgr 1 tiles such that the erosion behaviour of the W-r1 and W-r2 stripes on the tiles Bgr 1-1 and Bgr 1-2 are comparable, as seen from Figure 1(b).

The missing data points of the W-r2 curve of the tile Bgr 1-1 in Figure 1(b) are due to the delamination of the coating during the 2010/11 campaign. The damage is, however, rather localized: The measurements on the tile Bgr 1-2 were taken toroidally some $5 \mathrm{~mm}$ off the corresponding poloidal row on the tile Bgr 1-1, and with this small adjustment the worst-affected region could be avoided.

The results in Figure 1 can be explained by considering a rough surface being eroded in a step-by-step manner such that material is removed from plasma-inclined areas and deposited in valleys behind protruding hills, being then shadowed from further plasma contact [8]. As a result, the original surface will be largely modified, but this is not directly revealed by RBS since the ion beam is much larger than the typical size of the resulting surface features. Indirect evidence exists, though: In the case of the rough $\mathrm{W}$-r1 stripes, the peaks corresponding to tungsten were considerably broader than before the campaign and merged with the peaks from the graphite substrate.

SIMS depth profiles in Figure 2 show even more clearly the qualitative difference between the rough and smooth $\mathrm{W}$ stripes after their plasma exposure. These profiles have been recorded at $s \approx 1150 \mathrm{~mm}$ from the W-r1 and W-r2 stripes of the tile Bgr 1-2. For the smooth W-r2 stripe, a well-defined W coating (thickness $\approx 1.8 \mu \mathrm{m}$ ) is visible, as can be observed from the distinct minimum of the $\mathrm{C}$ signal, while in the case of the rough W-r1 stripe, the original coating has been replaced by an about $1.5-\mu \mathrm{m}$ thick surface layer, consisting of tungsten, boron, carbon, and deuterium. The long decay lengths of the $\mathrm{D}, \mathrm{B}$, and $\mathrm{W}$ profiles reflect the large roughness of the layer and sputtering of material from the edges of a SIMS crater.

Along with erosion and surface modifications, we noticed that some 5-10 times more boron and deuterium had been deposited on rough than on smooth coatings. This can be noticed in Figure 3 where the poloidal deposition profiles of the two elements on the different marker stripes of the tile Bgr 1-2 are presented; the curves for the tile Bgr 1-1 are very similar. The figure also shows the corresponding profiles for carbon which indicate a remarkable deposition peak on the W-r1 stripe. This result is, however, a bit deceiving since the contribution of the graphite substrate (see section 3.2) cannot be fully distinguished from that of the deposited layer. Nonetheless, all the curves in Figure 3 prove that deposition plays the largest role for the roughest surfaces.

\subsection{Surface morphology}

The ion-beam results discussed above can be understood by the different surface morphology of the rough and smooth marker stripes, as illustrated by the backscatter-electron SEM images in Figure 2. Figure 2(c) shows the surface of the W-r1 stripe on the tile Bgr 1-1 at $s \approx 1080 \mathrm{~mm}$ while the image in Figure 2(d) has been taken from the smoother W-r2 stripe, also at $s \approx 1080 \mathrm{~mm}$.

In the case of the rough W-r1 stripe, Figure 2(c) shows that the original coating has completely disappeared from some areas (thus appearing black), which are located around the tallest peaks on the surface. The eroded material is, however, largely re-deposited together with other elements behind the hills as one can see from the fringe patterns downstream of the black regions and slightly darker areas 
behind the fringes, which indicates deposition in surface depressions. For the W-r2 stripe (Figure 2(d)), less deposited material and less surface modifications can be observed. We can conclude that in the case of rough coatings, gross erosion is large but due to considerable (re)deposition, the overall effect in the macroscopic scale is reduced net erosion compared to smooth or as-milled coatings. One should, however, note that the total erosion is limited by the thickness of the $\mathrm{W}$ marker.

Confocal microscopy was used to determine the key roughness parameters for the regions W-r1, W-r2, and $\mathrm{W}-\mathrm{r} 3$. The results indicated that plasma exposure had made the coatings smoother on average but the effect had been the largest for the originally smoother stripes (4\% reduction for $\mathrm{W}-\mathrm{r} 1,40 \%$ for $\mathrm{W}$ r2 and W-r3). This supports the observations made in Figures 2(c) and (d).

\subsection{Effect of plasma-facing material}

Compared to tungsten, the other studied marker elements, $\mathrm{Al}, \mathrm{Cr}$, and $\mathrm{Mo}$, are eroded at considerably larger rates. Also re-deposition of the eroded material in the private-flux region below the OSP is larger for these elements. These results have been obtained from the tile Bgr 1-3 and they are collected in Figure 4. The curves in the figure indicate erosion being more intense for the lighter materials: For W $(Z=74)$, the net erosion rate is $0.03 \mathrm{~nm} / \mathrm{s}$ whereas Mo $(Z=42)$ had been eroded approximately 2-3 times faster and $\mathrm{Cr}(Z=24)$ 3-4 times faster. The whole Al layer was damaged because of its low melting point and, as a result, its nominal erosion rate was as large as $0.7 \mathrm{~nm} / \mathrm{s}$. The same tendency was observed also earlier but mainly in carbon-dominated machines, where the erosion rates were some 3-4 times higher than the results reported here [9].

\section{Conclusions}

In this article, the effect of surface roughness on the long-term erosion patterns of tungsten coatings was investigated in the OSP region of AUG. When the surface roughness was $R_{\mathrm{a}}=1.5 \mu \mathrm{m}$, the net erosion rate was $0.03 \mathrm{~nm} / \mathrm{s}$ but this value was reduced by a factor of $2-4$ as the roughness increased to $R_{\mathrm{a}}=5-6 \mu \mathrm{m}$. On the other hand, as the surfaces were smoother $\left(R_{\mathrm{a}}=0.4-0.8 \mu \mathrm{m}\right)$, net erosion was 2-4 times more intense. Our analyses showed that in the microscopic level, parts of the rough coatings had been completely eroded but most of the eroded material had been re-deposited together with boron, deuterium, and carbon on the shadowed sides of the most prominent surface features. On smoother surfaces, the deposited layers were much thinner and the surface structure also less modified.

Compared to other potential first-wall materials, we noticed that $\mathrm{W}$ was eroded 2-3 times less than Mo and 3-4 less than $\mathrm{Cr}$. In the case of $\mathrm{Al}$, the marker had been partly melted and badly damaged. In conclusion, surface morphology and deposition of low-Z impurities play important roles when comparing erosion measurements from different PFCs and from different tokamaks.

\section{Acknowledgments}

This work, supported by the European Communities under the contract of Association between Euratom and Tekes, was carried out within the framework of the European Fusion Development Agreement. The views and opinions expressed herein do not necessarily reflect those of the European Commission. The authors would like to thank G. Matern for assistance with SEM. 


\section{References}

[1] Philipps V 2011 J. Nucl. Mater., 415 S2

[2] Roth J et al 2008 Plasma Phys. Control. Fusion 50103001

[3] Kallenbach A et al 2005 Plasma Phys. Control. Fusion 47 B207

[4] Neu R et al 2009 Phys. Scr. T138 014038

[5] Maier H et al 2002 J. Nucl. Mater. 307-311 120

[6] Hakola A et al 2010 Plasma Phys. Control. Fusion 52065006

[7] Herrmann A et al 2009 Phys. Scr. T138 014059

[8] Schmid K et al 2010 Nucl. Fusion 50105004

[9] Federici G et al 2001 Nucl. Fusion 411967 


\section{Figure captions}

Figure 1. (a) Outer strike-point distribution during the 2010/11 experimental of campaign together with schematic illustration of the divertor (inset). (b) Poloidal erosion/deposition profiles of the different $\mathrm{W}$ marker stripes on the tiles Bgr 1-1, 1-2, and 1-3. Notice the delamination of the W-r2 stripe on the tile Bgr 1-1 around $s=1100-1150 \mathrm{~mm}$ and the stripe W-r3 of the same tile being shadowed during its exposure. (c) Re-deposition of $\mathrm{W}$ on the Mo and $\mathrm{Cr}$ markers of the tile Bgr 1-3.

Figure 2. (a,b) SIMS depth profiles of D, B, C, and W on the (a) W-r1 and (b) W-r2 stripe of the tile Bgr 1-2 at $s \approx 1150 \mathrm{~mm}$. (c,d) Backscatter-electron SEM images of the (c) W-r1 and (d) W-r2 stripe of the tile Bgr $1-1$ at $s \approx 1080 \mathrm{~mm}$.

Figure 3. Poloidal deposition profiles of (a) deuterium, (b) boron, and (c) carbon on the different W marker stripes of the tiles Bgr 1-2 and 1-3 (reference).

Figure 4. Poloidal erosion/deposition profiles of the different marker stripes on the tile Bgr 1-3. 


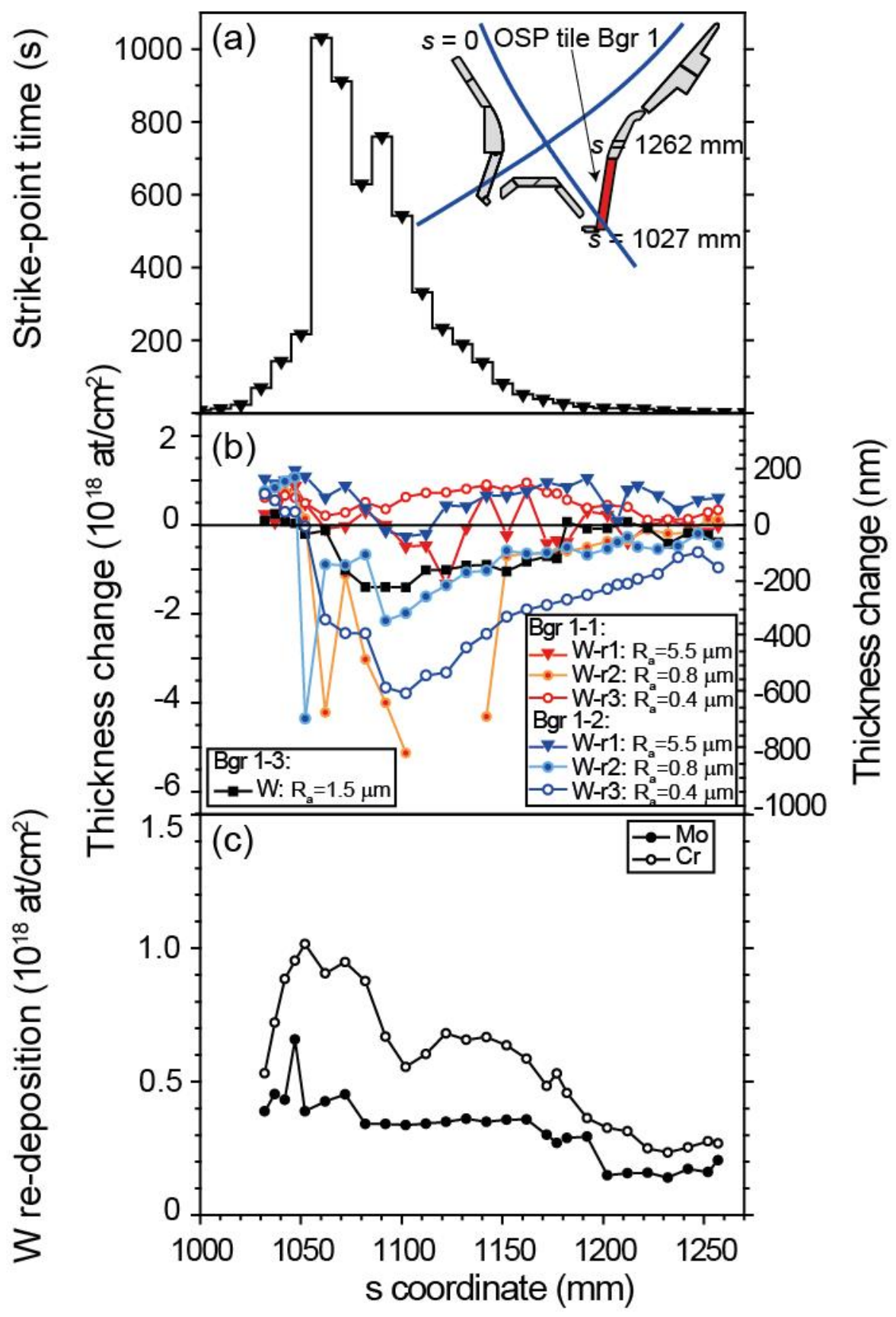

Figure 1 

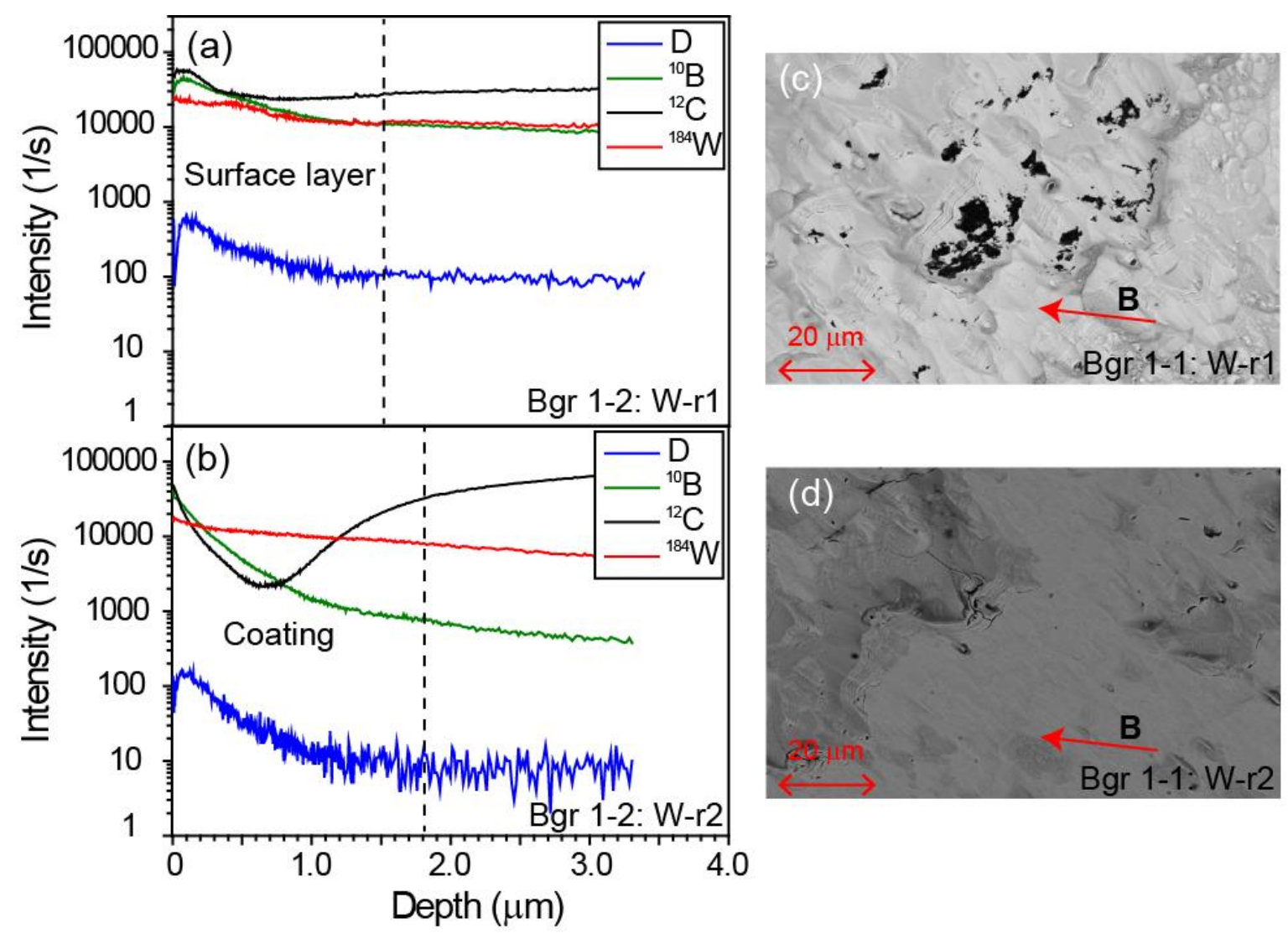

Figure 2 


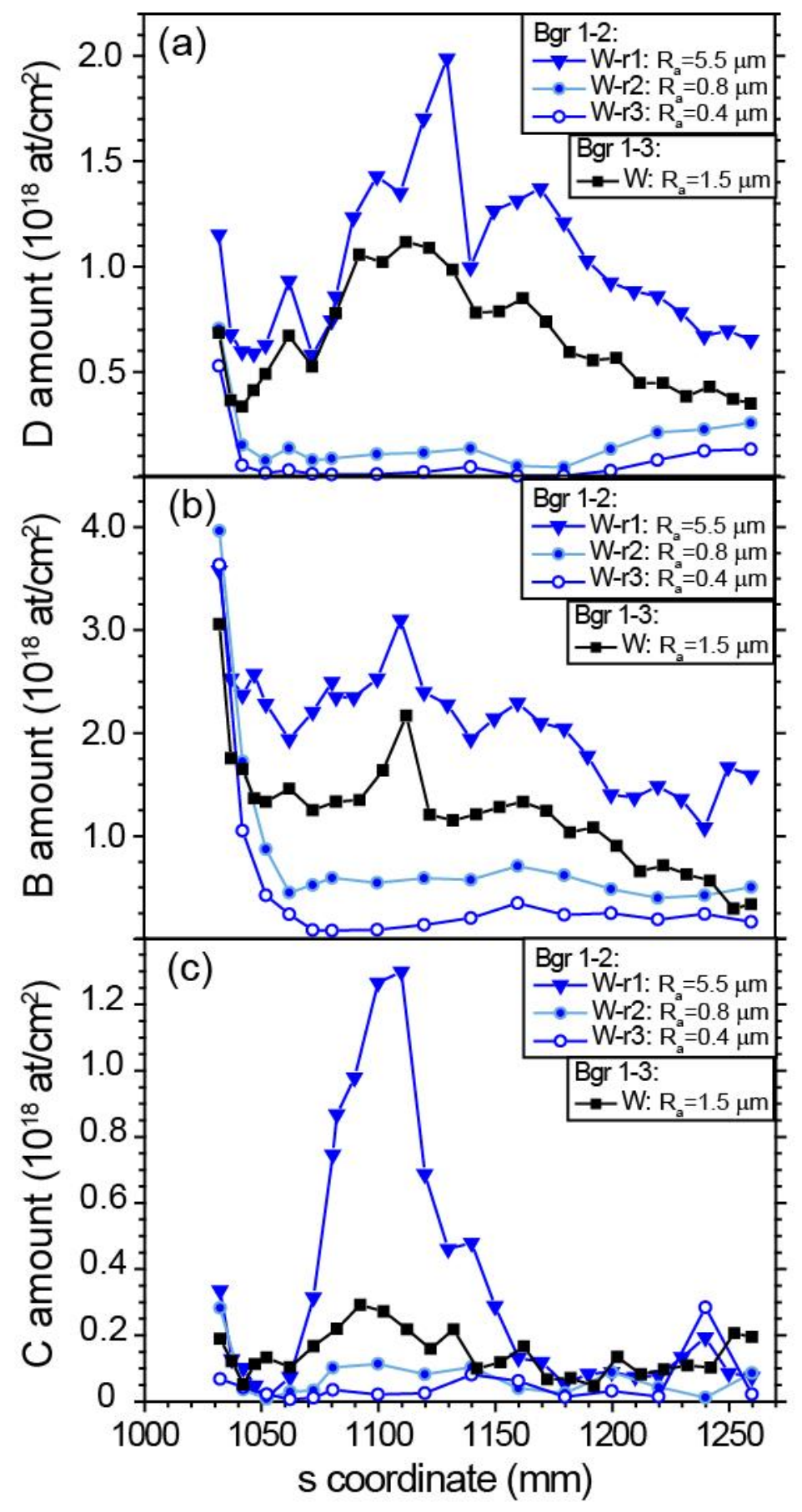

Figure 3 


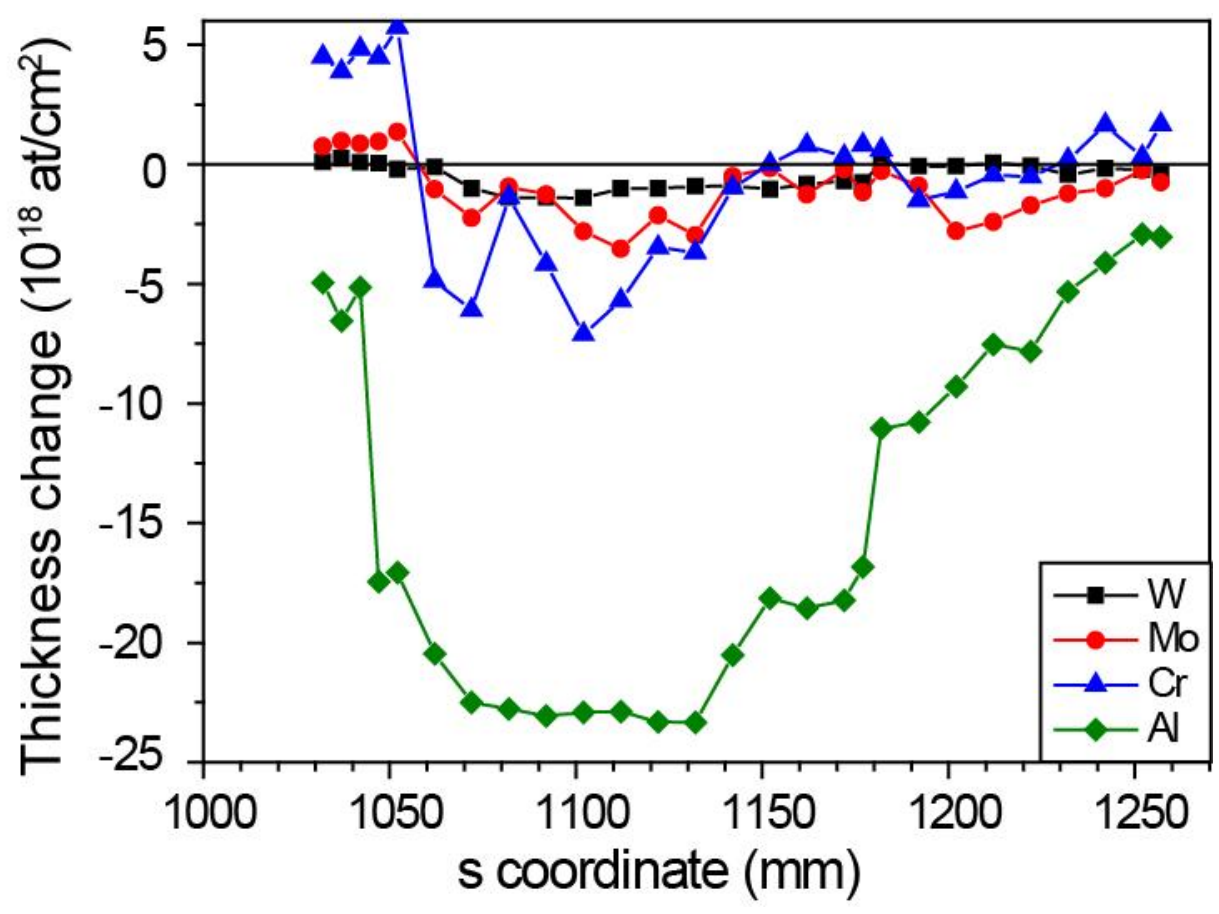

Figure 4 